\title{
Greedy segmentation based diabetic retinopathy identification using curvelet transform and scale invariant features
}

\author{
Pelin Görgel \\ Department of Computer Engineering, Istanbul University-Cerrahpasa, Istanbul 34320, Turkey \\ Corresponding Author: paras@istanbul.edu.tr
}

Submitted: 26/12/2019

Revised: $\quad 15 / 11 / 2020$

Accepted: 22/11/2020

\begin{abstract}
Diabetic retinopathy (DR) is the major reason of vision loss in the active population. It can usually be prevented by regulating the blood glucose and providing a timely treatment. DR has clinical features recognized by the experts including the blood vessel area, exudates, neovascularization, hemorrhages, and microaneurysm. Because DR has some varieties and complexities due to its geometrical and haemodynamic features, it is hard and time-consuming to detect DR in manual diagnosis. In Computer Aided Diagnosis (CAD) systems, the features of DR fundus images are detected using computer vision techniques. In this paper, a CAD system is proposed, which distinguishes automatically whether the fundus is normal or it suffers from diabetic retinopathy disease. As preprocess morphological operations like filtering, opening, and dilation are applied to the images firstly, then, Optic Disk (OD) segmentation is implemented using Greedy algorithm. Because of the intensity of an OD is similar to some DR intensities, OD regions are removed from the fundus images for an accurate feature extraction. The features extracted with Curvelet Transform (CT) and Scale Invariant Feature Transform (SIFT), respectively, are concatenated to provide a feature set that defines the fundus data optimally. Finally, the feature set is given to the Support Vector Machines (SVM), K-Nearest Neighborhood (KNN), and Naïve-Bayes (NB) classifiers for the DR identification purpose. The proposed method achieves the highest accuracy and sensitivity as $92.8 \%$ and $97.6 \%$, respectively, with SVM and specificity as $92.5 \%$ with KNN classifier.
\end{abstract}

Keywords: Diabetic Retinopathy, Greedy Segmentation, Curvelet Transform, Scale Invariant Feature Transform, Support Vector Machines.

\section{INTRODUCTION}

Diabetes occurs in the case of glucose increase in blood. If this disease continues for a long time, it damages the blood vessels irreversibly. Diabetic patients may also suffer from vision loss, kidney failure, and nerve damage. They have even a high risk of heart attack and stroke (Shanthi et al., 2019). The World Health Organization (WHO) supposes that diabetes will be the seventh leading fatal disease (Sarwar et al., 2010). The number of diabetic people has risen from 108 million in 1980 to 422 million in 2014. Also, the prevalence of diabetic patients above 18 years of age arised from $4.7 \%$ to $8.5 \%$ (Whiting et al., 2011). Diabetic retinopathy is originated from diabetes complications that affect ocular perceivers and veins of light-delicate tissues (Sayin et al., 2015 \& Abed et al., 2019). Sudden vision loss, blurred vision, eye flashes, and floaters (Nadeem et al., 2019) are the common symptoms of DR. As blood level increases, it damages the blood vessels in retina and causes a possible blood leakage into the eye. When a leakage occurs, the brain leads an abnormal growth of new blood vessels to control the situation (Alipour et al., 2012). 
The disease severity is defined according to the magnitude of abnormalities such as microaneurysms, hemorrhages, exudates, and neovascularization. The stages of DR are categorized as mild, moderate, and severe due to their clinical, geometrical, and haemodynamic features. The microaneurysms are blood vessels deformations, while the hemorrhages are blood leakages from the damaged capillaries, and generally, they are dark red spots (Mookiah et al., 2013; Zhang, 2008). Hard exudates, which are white colored, sharp, and circular, reside on the retina around vessels. Soft exudates occur in case of occlusion of arteriole, and they seem as fluffy white lesions. A normal left and right eye fundus image pair is shown in Fig. 1, and anormal fundus images with DR are demonstrated in Fig. 2. Early detection and treatment are essential in preventing visual degradation. DR can efficiently be diagnosed at early levels by the help of computer based automated detection.
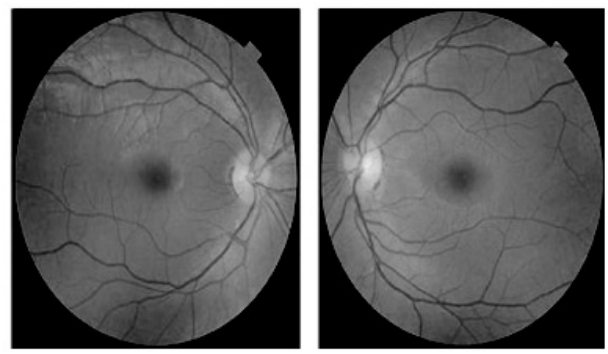

Figure 1. A normal fundus of left and right eye (Nadeem et al., 2019).

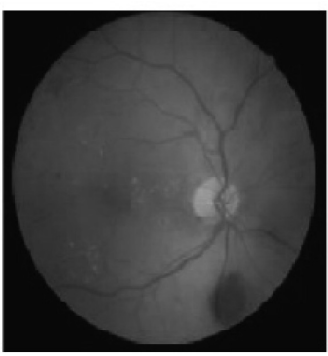

a)

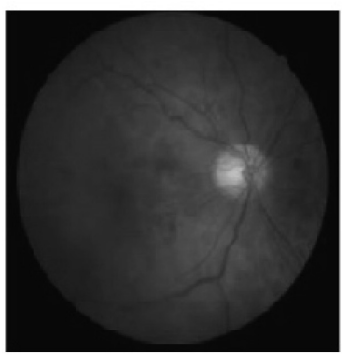

b)

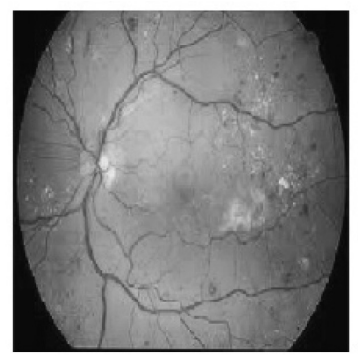

c)

Figure 2. DR fundus images: a) Presence of microaneurysms and soft exudates-mild stage. b) Presence of microaneurysms in a bigger amount as compared to mild stage-moderate stage. c) Presence of hard exudates and microaneurysms-severe stage (Doshi et al., 2016).

The motivation of this paper is to provide an automatic diagnosis of DR using image classification. Firstly, OD is segmented and removed from the fundus images. In this stage, the preprocessing methods such as morphological openning, dilation, and structure elements are used prior to Greedy segmentation. The extracted optic disk is neutralised by changing the disk pixel intesities with the mean intensity of nearest local neighbourhood. Next, feature extraction, which is an essential for classification, is applied to OD eliminated images including several exudates, hemorrhages, and microaneurysms. In this study, in addition to the Curvelet Transform, Scale Invariant Feature Transform (SIFT), which utilizes Difference of Gaussians to detect blobs of various sizes, is implemented. The combined CT-SIFT feature set is given to the Support Vector Machine (SVM), which is an efficient data classifier. The main contributions of this study are summarized as follows.

A fundus DR identification methodology based on both CT and SIFT features is proposed. The method improves a satisfying classification accuracy of $92.8 \%$ in distinguishing the normal fundus from DR fundus images when tested against two well-known public datasets: the Diabetic Retinopathy Database DIARETDB1 and DIARETDB0. The morphological preprocessing and Greedy segmentation help reach initial OD contours automatically instead of defining them manually. Greedy method makes calculations in each iteration until it reaches the optimum OD contour. After ODs are extracted, they are eliminated because of the similar intensity between OD and DR soft or hard 
exudates. The purpose is not to allow this similarity to reduce the overall classification accuracy via manipulating the feature extraction.

For the feature extractor, Curvelet Transform is harnessed as it is efficient in representing the nature of the curvature tumor shapes due to its convenience to curve based objects. Also, a SIFT feature set is combined to CT coefficients to have an accurate definition of fundus data, which contains important diagnostic information.

\section{RELATED WORK}

For the identification of diabetic retinopathy, several computer aided diagnosis systems have been proposed recently. Decencière et al. (2013) used retinal image information and data such as age, weight, or diabetes history belonging to the patients to detect DR. The authors utilized data mining with image quality metrics, the presence of exudates, microaneurysms, hemorrhages, and diabetes demographic information. Lachure et al. (2015) used Support Vector Machines and KNN to distinguish DR fundus images. Preprocessing and morphological operations were performed to find the microaneurysms, and then, identical features were extracted to classify normal and DR images. According to the results, SVM showed better performance than the KNN classifier. In the study of Asha et al. (2015), machine learning methods were used to find the absence or presence of exudates in retinal images. Edge strength, centroid, standard deviation, and mean features were extracted for each fundus lesion. Colas et al. (2016) and Gulshan et al. (2016) used Deep Neural Network (DNN) to identify referable DR. In the study of Adem (2018), basic preprocessing methods like adaptive histogram equalization and Canny edge detection were used for visual enhancement. For the optic disk segmentation, circular Hough transformation was implemented. After that, Convolutional Neural Networks $(\mathrm{CNN})$ based system was developed to detect the exudates automatically in the retinal images. The images were trained with CNN and classified as exudated and exudate-free. Osareh et al. (2002) classified the normal and abnormal fundus images using Fuzzy C-Means (FCM) algorithm with an accuracy of 90.1\%. Acharya et al. (2009) utilized the blood vessels, exudates, and hemorrhages morphological features using SVM classifier, and their method demonstrated a classification accuracy of $85.9 \%$, sensitivity of $82 \%$, and specificity of $86 \%$. Harangi and Hajdu (2014) firstly identified suspicious regions containing fundus abnormality due to DR with a gray scale morphology-based method. To extract the boundaries of the regions, a complex active contour-based method was used. A region-wise classifier was applied to remove the false exudate candidates. Several region-based features constituted a subset to train a NB classifier optimized further by an adaptive boosting technique. Their method accomplished DR identification accuracy of 82\% using DIARETDB1 dataset. Koh et al. (2017) used 2D-Continuous Wavelet Transform (CWT) for the decomposition of fundus images. Energy features and entropies such as Yager, Renyi, Kapoor, Shannon, and Fuzzy were extracted from the decomposed images. Adaptive synthetic sampling approach was performed to balance the normal and abnormal datasets next. Then, the extracted features were optimized using Particle Swarm Optimization (PSO). In the training stage, the Random Forest (RF) classifier was applied using stratified 10-fold cross validation. Alshayeji et al. (2017) subjected the images to a smoothing process called anisotropic diffusion for DR identification. They used an edge detection algorithm inspired by the gravitational law. In the study of Abed et al. (2016), swarm intelligence algorithms such as artificial bee colony and particle swarm optimization were applied to increase the detection accuracy. Before detection, a preprocessing step involving multiple stages of background subtraction, median, and mean filtering was implemented. Acharya et al. (2011) identified DR using Higher Order Spectra (HOS) texture analysis to analyze the textural characteristics of the fundus images. HOS based features were extensively used in SVM classifier for automatic diagnosis of normal and glaucoma classes. Agurto et al. (2010) proposed amplitudemodulation-frequency-modulation (AM-FM) texture feature extraction method. The system accuracy was calculated according to distance method. This method achieved accuracy of $92 \%$, sensitivity of $90 \%$, and specificity of $80 \%$. Ram and Sivaswamy (2009) focused on clustering based segmentation of exudates and extracted multispace and color-space features. The accuracy was up to $89.6 \%$ and gave positive prediction value of $87 \%$. Yafen et al. (2013) proposed a new method including techniques such as image enhancement, morphological image processing, and texture analysis. For the classification, SVM was preferred, and the system accuracy, the sensitivity, and the specificity were obtained as $89 \%, 90 \%$, and $95 \%$, respectively. In this study, an effective methodology is implemented, which achieves a better DR identification accuracy. 


\section{PROPOSED METHODOLOGY}

In a fundus image, OD can be seen clearly due to its distinctive intensity. If ODs are taken into account in feature extraction, it would manipulate the seperation of the normal and DR (anormal) fundus images. The reason is that ODs are brighter and cover more area than the other fundus regions. Therefore, the first goal in the proposed system is to segment the ODs correctly and then eliminate them (Fig. 3). Using only threshold methods does not work in OD extraction, since OD and exudates both have bright intensities. Hence, before Greedy algorithm, a set of enhancement operations, namely, preprocessing, is implemented. Then, initial contour sets are obtained for each fundus using a thresholding step. For the next, these contour sets are given to Greedy algorithm as initial contours to extract the ODs exactly. Greedy segmentation tries to make the choice that always seems to be the best at that moment. It makes a locally optimal choice, hoping that this choice will obtain a globally optimal solution. To calculate the initial contours, Greedy algorithm helps an automatic OD contour definition instead of drawing them manually for each fundus.

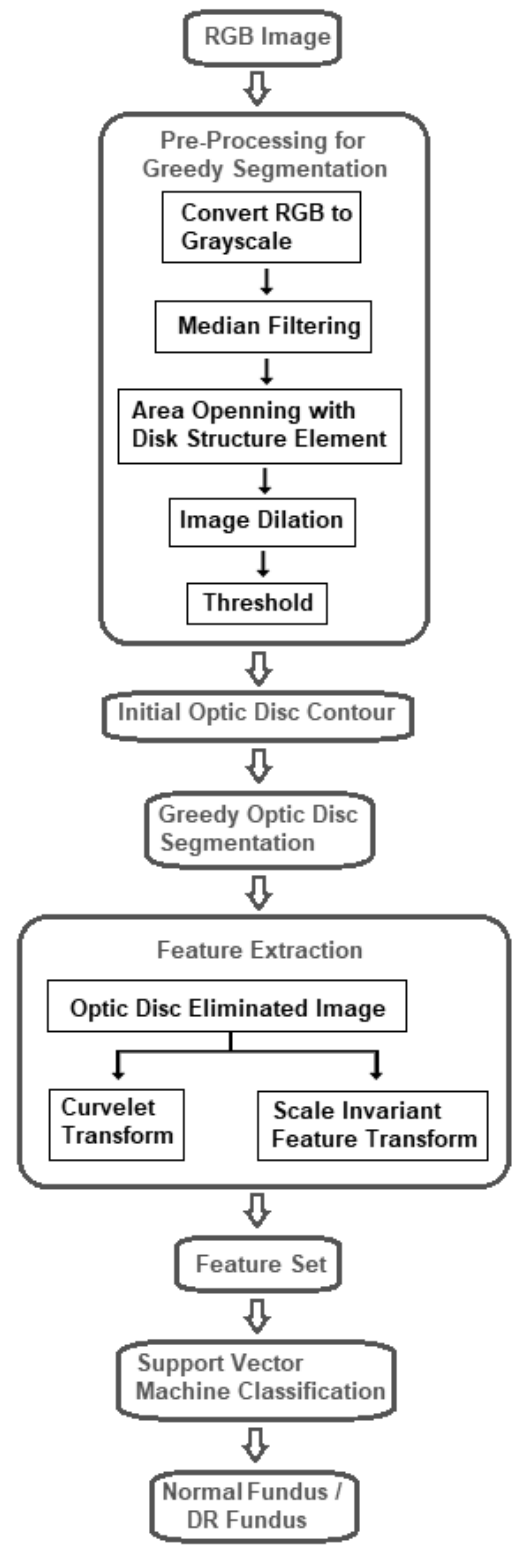

Figure 3. Flowchart of the proposed methodology. 
Greedy method takes these sets and makes a series of calculations regarding the neighbour pixels in each iteration until it reaches the optimum OD contour. After an optic disk is extracted, it is eliminated by assigning the mean of the closest pixels around the disk to the whole disk intensity. After that, it is aimed to extract the distinctive features of normal and DR fundus images, which have abnormal parts like exudates, microaneurysms, and hemorrhages using curvelet cransform, in addition to scale invariant feature transform. The obtained feature set for each fundus is given to the Support Vector Machines (SVM). SVM classifies all fundus images as normal or DR automatically. To sum up, the proposed methodology consists of four steps: preprocessing, greedy segmentation, SIFT, and curvelet transform based feature extraction and classification.

\section{Preprocessing for the Initial Optic Disk Contour Extraction}

\section{Median Filtering for Noise Removal}

Median filter is used to reduce the noise in the grayscale fundus images caring to keep the fine details in the images. A median filter takes into account the neighborhoods to adjust each pixel's value. The neighbor pixels are sorted, and the value in the middle of an array is taken as the new value. If there are even numbers of pixels in the region of interest, the average of the two pixels in the middle is used as the median (Weiss, 2006; Görgel et al., 2018). In equation (1), $w$ is the required neighborhood, $[m, n]$ is the central location in the image $x$, and $y$ is the median. Area opening follows the noise removal substep.

$$
y[m, n]=\operatorname{median}\{x[i, j],(i, j) \in w\}
$$

\section{Area Opening Using Disk Structure Element}

In mathematical morphology, special set families, called structuring elements, can be adjusted according to the need in terms of size, shape, and orientation. In this study, a disk structure element, which is a binary mask generated for morphological opening, is used because of the circular shape of optic disks. The morphological opening operator consists of two operators, erosion and dilation, respectively. The combination of these operators behaves as a shape filter and removes image parts smaller than the structure element. In equation (2), the opening of a set $X$ by a structure element $B$ is $O_{x}$ (Nadeem et al., 2019):

$$
O_{x}=X \circ B=(X \ominus B) \oplus B
$$

\section{Image Dilation}

Dilation is simply the translation of a pixel value. The translation of $X$ by $p \in E$ is the set $X_{p}=\{x+p \mid x \in X\}$. Here, $X$ is a binary image of a subset $E$, and $p$ is the translation vector. Dilation enlarges the pixel $x$ by set $B$ (Gonzalez et al., 2002). $\delta_{B}(X)$ represents morphological dilation of image $X$ using $B$ structure element in equation (3).

$$
\delta_{B}(X)=X \oplus B=\cup_{b \in B} X_{b}=\cup_{x \in X} B_{x}=\{x+b \mid x \in X, b \in B\}
$$

After preprocessing, the images get ready for the Greedy segmentation.

\section{Greedy Optic Disk Segmentation}

Snakes (active contours) (Kass et al., 1988) are a different approach of feature extraction. A snake is a set consisting of points, and it aims to enclose a target feature. It is a bit like using a balloon to match a shape; the balloon is placed inside the shape. By blowing up the balloon and making it larger, the shape fits the target shape when it stops enlarging. By this manner, an initial contour is placed inside the target feature and is then evolved so as to enclose it. 
Snakes method is an energy minimization process. The target feature is a minimum of a suitably formulated energy functional. This functional includes properties that control the way the contour can stretch and curve, as well as edge information. Therefore, a snake represents a compromise between the image properties such as edge magnitude and the snake's own properties such as bend and stretch ability. The energy functional is the addition of a function of the contour's internal energy, the constraint energy, and the image energy, which are denoted by $W_{\text {int }}, W_{\text {con }}$, and $W_{\text {image }}$ respectively. These are the functions of the snake points set $v(s)$ including $x$ and $y$ coordinates of the points in the snake. The energy functional is the integral of these functions of the snake, given that $s \in[0,1]$ is the normalized length around the snake. The energy functional $W_{\text {snake }}$ is

$$
W_{\text {snake }}=\int_{s=0}^{1} W_{\text {int }}(v(s))+W_{\text {image }}(v(s))+W_{\text {con }}(v(s)) d s
$$

where $W_{\text {int }}$ controls the arrangement of the snake points, $W_{\text {image }}$ evaluates low-level features like edge points, and $W_{\text {con }}$ controls the snake evolution. The minimum $W_{\text {snake }}$ value is sought to find a better match to the target feature. Greedy algorithm (Williams et al., 1992) implements the energy minimization by a specified initial contour. It evolves the snake iteratively using local neighborhood around the contour points to select new points having lower snake energy. At each iteration, all contour points are evolved, and the process is actually repeated for the first contour point (Nixon et al., 2002). The discrete energy functional $W_{\text {snake }}$ can be rewritten as in equation (5). $W_{\text {edge }}$ is the magnitude of the Sobel edge operator at point $x, y$.

$$
W_{\text {snake }}(s)=k_{1}(s)\left|\frac{d v_{s}}{d s}\right|^{2}+k_{2}(s)\left|\frac{d^{2} v_{s}}{d s^{2}}\right|^{2}+k_{3}(s) W_{\text {edge }}
$$

The first- and second-order differentials are approximated for each point in the local neighborhood of currently selected contour point. As demonstrated in equation (5), each contour point has its own $k_{1}, k_{2}$ and $k_{3}$ parameter values. The first-order differential is the modulus of the difference with the average spacing of contour points and the Euclidean distance between the currently selected point $v_{s}$ and the next contour point as seen in equation (6). $k_{1}$ parameter controls the spacing between the contour points. $x$ and $y$ coordinates of the adjacent snake point are $x_{s+1}$ and $y_{s+1}$. The coordinates of the currently investigated point are $x_{s}$ and $y_{s}$. Let $v_{s+1}, v_{s-1}$ and $v_{s}$ be the next contour point, previous contour point, and currently investigated snake point, respectively. The second-order differential is an estimate of the curvature between the next and previous contour points as demonstrated in equation (7).

$$
\begin{aligned}
& \left|\frac{d v_{s}}{d s}\right|=\left|\sum_{i=0}^{S-1} \frac{\left\|v_{i}-v_{i+1}\right\|}{s}-\left\|v_{s}-v_{s+1}\right\|\right| \\
& \left|\frac{d^{2} v_{s}}{d s^{2}}\right|^{2}=\left|\left(v_{s+1}-2 v_{s}+v_{s-1}\right)\right|^{2}
\end{aligned}
$$

The pseudocode of the Greedy algorithm is demonstrated in Fig. 4. In the algorithm used in this study, $s$ and $S$ represent the contour set and the number of set points, respectively. $N$ represents the number of neighbours, which is 8 due to $3 \times 3$ neighbourhood. The calculate function takes the parameter of $x$ and $y$ coordinates of the current point. This function calculates minimum energy using equations (5) and (6). Also greedy function takes $k_{1}, k_{2}$ and $k_{3}$ parameters. $k_{1}$ controls the spacing on the contour points, $k_{2}$ arranges the curvature, and $k_{3}$ arranges the contribution of edge information in the image. After Greedy segmentation, the extracted ODs are eliminated from the fundus images. For the next step, CT is utilized for the first part of feature extraction. 


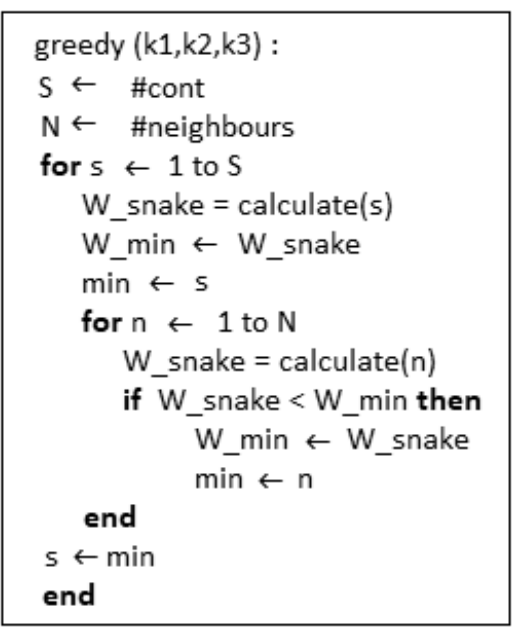

Figure 4. The Greedy algorithm.

\section{Curvelet Transform based Feature Extraction}

Curvelet Transform introduced by Candès et al. (2000) uses a geometric and directional wavelet that splits the whole frequency domain into multidirection and multiscale as demonstrated in Fig. 5. As seen in equation (8) CT decomposes the image $I$ into curvelet coefficients $T_{j, l, k}$, which can be defined by an inner product. $\theta_{j, l, k}$ is the curvelet basis function, and $j, l, k$ represent scale, orientation, and location, respectively.

$$
T(j, l, k)=\left\langle I, \theta_{j, l, k}\right\rangle=\int_{\mathbb{R}^{2}} I(x, y) \overline{\theta_{J, l, k}} d x d y
$$

The continuous curvelet transform (CCT) is represented by polar coordinates $r$ and $\psi$ along with $x$ spatial variable and $w$ frequency domain variable (equation (9)). In CT, the image is represented with various windows at different scales and orientations. The frequency window $U_{j}$ in Fourier domain can be defined as (Nayak et al., 2017)

$$
U_{j}(r, \psi)=2^{-\frac{3 j}{4}} W\left(2^{-j} r\right) V\left(\frac{2\left\lfloor\frac{j}{2} \mid \psi\right.}{2 \pi}\right)
$$

In equation (9), $W(r)$ and $V(t)$ are radial and angular windows, respectively, while $\lfloor j / 2\rfloor$ indicates the integer part of $j / 2$.

The discrete curvelet transform (DCT) decomposes the image $I$ into curvelet $S$ levels, with $L_{s}$ directions and $K_{s, l, 1} \times K_{s, l, 2}$ spatial shifts for each direction as seen in Equation (10). $\theta_{j, l,(k 1, k 2)}$ is the curvelet element on level $s$ and spatial shift $k_{1}, k_{2}$.

$$
I(x, y)=\sum_{j=1}^{J} \sum_{l=0}^{L_{j-1}} \sum_{k_{1}=0}^{K_{j, l, 1}-1} \sum_{k_{2}=0}^{K_{j, l, 2}-1} T_{j, l,(k 1, k 2)} \theta_{j, l,(k 1, k 2)}(x, y)
$$

In equation (11), $U_{s}$ is the polar wedge, and in equation (12), $\theta_{s, l,(k 1, k 2)}$ is defined by using Fourier transform.

$$
\begin{aligned}
& \theta_{S, 0,(k 1, k 2)}(x, y)=U_{S}(x, y) e^{-2 \pi i\left(\frac{k_{1} x}{K_{S, 0,1}}+\frac{k_{2} x}{K_{S, 0,2}}\right)}, \\
& \theta_{S, l,(k 1, k 2)}=S_{\psi_{l}}^{T} \theta_{S, 0,(k 1, k 2)}
\end{aligned}
$$


Continuous Time

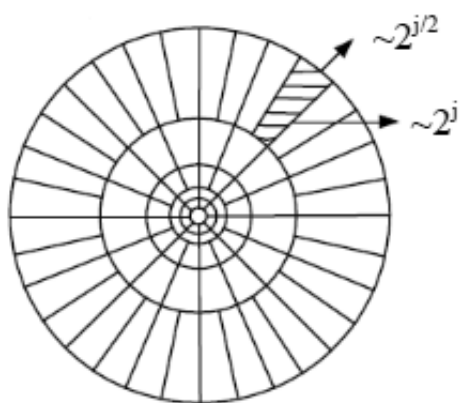

Digital

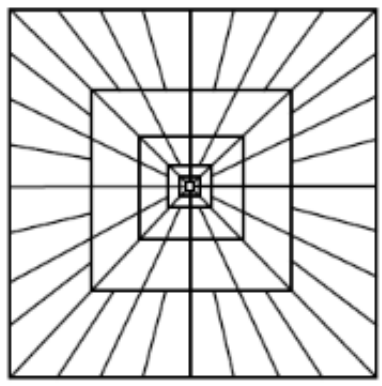

Figure 5. Multidirection and multiscale in curvelet transform (Shang et al., 2008).

In this study, the curvelet coefficients are used as image features combined with scale invariant features. For the next step, SIFT is implemented for the second part of feature extraction.

\section{Scale Invariant Feature Transform}

Scale invariant feature transform (SIFT) is a frequently used feature extraction method in pattern recognition applications (Qin et al., 2012). Being scale and orientation invariant is the main benefit of the SIFT algorithm. It is utilized in digital images to detect and extract local features (Lowe, 1999). SIFT produces a large set of features from the images by decreasing local variation errors in feature matching. It is able to identify objects in case of partial occlusion among clutter, and it is processed in four steps: scale-space extrema detection, keypoint localization, orientation assignment, and keypoint descriptor.

- Scale-space extrema detection: keypoints are the points of interest in an image. In this stage, the Gaussian filter for convolution in different scales and the difference of successive Gaussian-blurred images (Mordvintsev et al., $2013)$ are used. The difference of Gaussians (DoG) image $D(x, y, \sigma)$ at multiple scales is given by

$$
D(x, y, \sigma)=L\left(x, y, k_{i} \sigma\right)-L\left(x, y, k_{j} \sigma\right)
$$

In equation (14), $I(x, y), L(x, y, k \sigma), G(x, y, k \sigma), k_{i} \sigma$ and $k_{j} \sigma$ represent the raw image, convolution of the raw image, Gaussian blur, and $i$ and $j$ scales, respectively (Görgel, 2019). Convolution of the images in different scales is implemented to detect scale space extrema as in equation (14).

$$
L(x, y, k \sigma)=G(x, y, k \sigma) * I(x, y)
$$

- Keypoint localization: many endpoints that are not actually keypoints may be detected. Such ambiguous points should not be included in the keypoints set. Hence, with the help of some filters and thresholds, low contrast and weak keypoints are eliminated (Lowe, 1999). $D(x)$ represents the Taylor expansion in equation (15), and $X$ is the computed offset in equation (16).

$D(x)=D+\frac{\partial D^{T}}{\partial x} x+\frac{1}{2} x^{T} \frac{\partial^{2} D}{\partial x^{2}} x$

$X=(x, y, \sigma)^{T}$

- Orientation assignment: the magnitude and direction of each keypoint orientations are calculated in this step (Panchall et al., 2013). This is done for all neighboring pixels of each keypoint in the image. A region is selected around each keypoint candidate, and these values are obtained on the softened image $L$. The orientation magnitude $m(x, y)$ and the orientation angle $\theta(x, y)$ are given by equations (17) and (18), respectively (Lowe, 2004). 


$$
\begin{aligned}
& \sqrt{(L(x+1, y)-L(x-1, y))^{2}+L(x, y+1)-L(x, y-1)^{2}} \\
& \theta(x, y)=\tan ^{-1} \frac{(L(x+1, y)-L(x-1, y))}{(L(x, y+1)-L(x, y-1))}
\end{aligned}
$$

- Keypoint descriptor: the descriptors around the keypoints are determined with their orientation direction and magnitudes. The identification of the descriptors that take place in a $4 \times 4$ area with $16 \times 16$ neighborhood of the keypoint is given in Fig. 6 . In these subareas histogram values are obtained by adding the orientation magnitudes corresponding to the same region within 45-degree slices. As a result, SIFT feature vector is of size 128 since each of $4 \times 4$ squares has 8 descriptors (Karaköse et al., 2017).

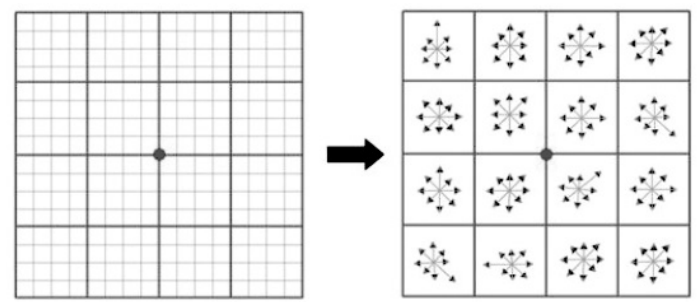

Figure 6. Detection of interest point descriptors (Karaköse et al., 2017).

After SIFT is implemented, the feature matrix is given to the SVM classifier to identify DR in fundus images.

\section{Support Vector Machines}

Cortes et al. (1995) introduced Support Vector Machines as a classification method, which aims to determine a hyperplane as the decision surface via maximizing the negative and positive data sample separation margin. The relation in equation (19) should be maximized to obtain an optimum SVM classifier boundary in given label pairs $\left(\mathrm{x}_{\mathrm{i}}, \mathrm{y}_{\mathrm{i}}\right) . P$ is defined as a positive parameter to control the tradeoff between the nonseparable points and the complexity (Cortes et al., 1995). $L(c), l, \mathrm{~K}\left(\mathrm{x}_{\mathrm{i}}, \mathrm{x}_{\mathrm{j}}\right)$, and $c_{i}$ are Lagrange function of all samples, the sample number, SVM kernel, and Lagrange coefficient, respectively, in equation (19). In equation (20), $w$ is the margin, and $\xi_{\mathrm{I}}$ is the slack variable to be used to relax the canonical hyperplane equation's constraints.

$$
\begin{aligned}
& L(c)=\sum_{i=1}^{l} c_{i}-\frac{1}{2} \sum_{i, j=1}^{l} y_{i} y_{j} c_{i} c_{j} K\left(x_{i}, x_{j}\right), \quad 0 \leq c_{i} \leq P \\
& w=\sum_{i=1}^{N} c_{i} y_{i} x_{i}, \quad c_{i}\left[y_{i}\left(w^{T} x_{i}+b\right)-1+\xi_{\mathrm{I}}\right]=0
\end{aligned}
$$

\section{Optic Disk Elimination}

Optic disk wholly seems brighter than the background in fundus images. In this study, ODs in both DR and healthy fundus images are eliminated via assigning a local neighborhood mean intensity value to the OD pixels due to similar intensity between ODs and DR exudates. This similarity would manipulate feature extraction and reduce the overall classification accuracy. In the proposed study, Greedy algorithm is used to segment the ODs before the elimination. The essential point is to determine the initial disk contour, which is given to Greedy algorithm as an input. In case of an accurate initial contour detection, the Greedy algorithm performs well and provides the correct optic disk contour. To achieve this task, a preprocessing stage including median filtering, area opening with disk structure element, and image dilation is implemented. Thresholding is applied to obtain the initial optic disk contour after preprocessing. OD is eliminated by assigning the mean of the closest pixels around the disk to the whole disk intensity. 
The proposed method is evaluated on DIARETDB0 (Kauppi et al., 2006) and DIARETDB1 (Kauppi et al., 2007), which are publicly available standard databases. The results are achieved with 125 color fundus images, of which 85 contain either hard exudates, soft exudates, microaneurysms, or hemorrhages and 40 are considered as normal containing no signs of DR. The images in the databases were captured using the same fifty-degree field-of-view digital fundus camera. The eye image with DR in Fig. 7 (a) has hard exudates signed with a yellow arrow. Fig. 7 (f) and 7 (h) demonstrate Greedy based optic disk contour detected and optic disk eliminated fundus images, respectively. Fig. 7 (c) is the preprocessed fundus, and Fig. 7 (d) is the obtained initial contour for Greedy algorithm. Eye image with DR in Fig. 8 (a) has hemorrhages signed with a yellow arrow. Fig. 8 (f) and 8 (h) demonstrate Greedy based optic disk contour detected and optic disk eliminated fundus images, respectively. Fig. 8 (c) is the preprocessed fundus, and Fig. 8 (d) is the obtained initial contour for Greedy algorithm. Additionally, Fig. 9 (a) represents a normal fundus image. Optic disk elimination is also implemented for normal fundus. Fig. 9 (f) and 9 (h) demonstrate Greedy based optic disk contour detected and optic disk eliminated fundus images, respectively. Fig. 9 (c) is the preprocessed fundus, and Fig. 9 (d) is the obtained initial contour for Greedy algorithm.

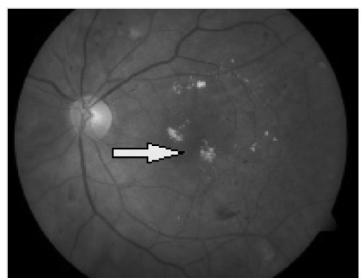

a)

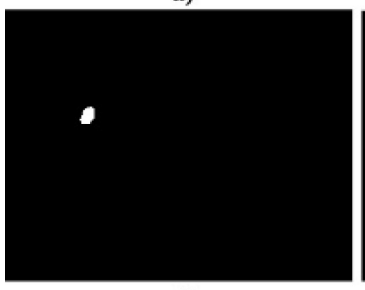

d)

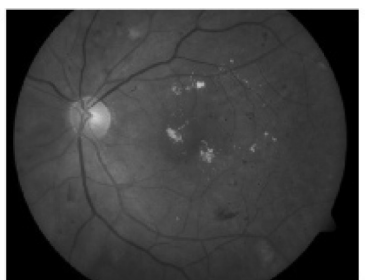

b)

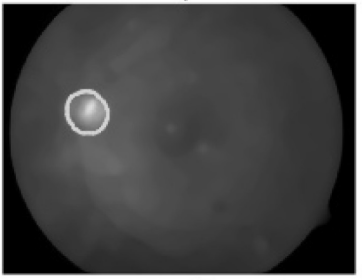

e)

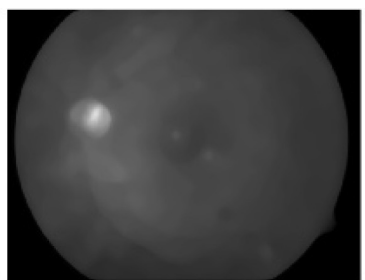

c)

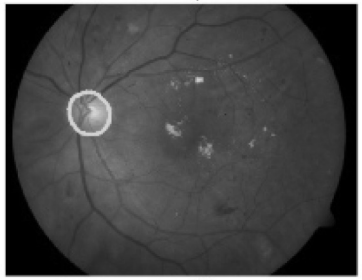

f)

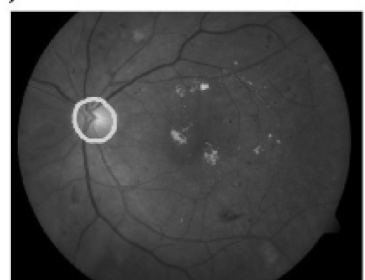

g)

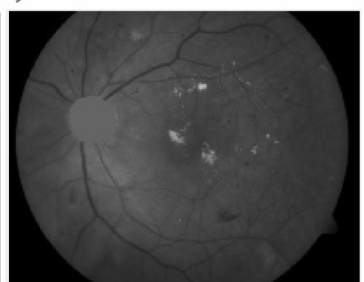

h)

Figure 7. (a) Original DR fundus with exudates signed with a yellow arrow. (b) Gray level fundus. (c) Preprocessed fundus. (d) Initial OD contour. (e) OD contour segmented with Greedy algorithm. (f) OD contour on original fundus. (g) OD contour on gray level fundus. (h) OD eliminated fundus. 


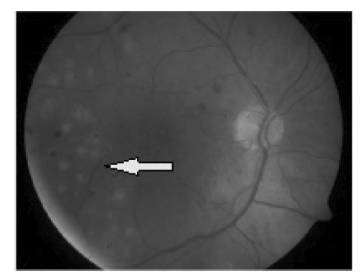

a)

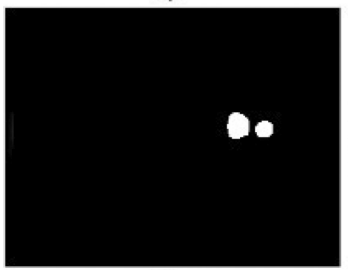

d)

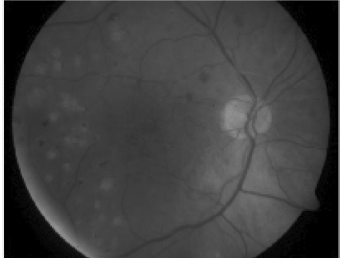

b)

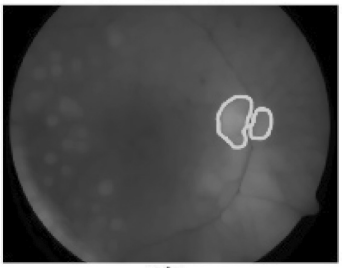

e)

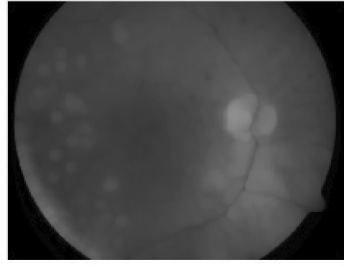

c)

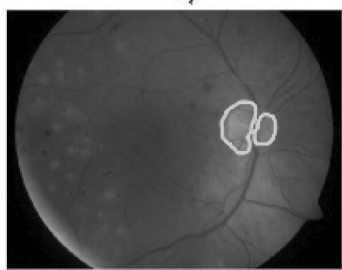

f)

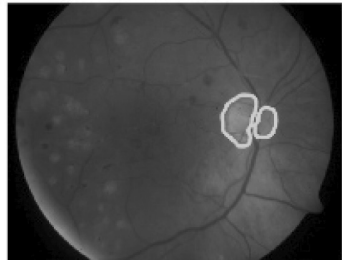

g)

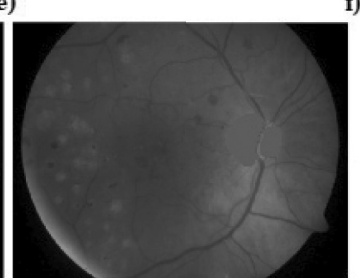

h)

Figure 8. (a) Original DR fundus with hemorrhages signed with a yellow arrow. (b) Gray level fundus.

(c) Preprocessed fundus. (d) Initial OD contour. (e) OD contour segmented with Greedy algorithm. (f) OD contour on original fundus. (g) OD contour on gray level fundus. (h) OD eliminated fundus.

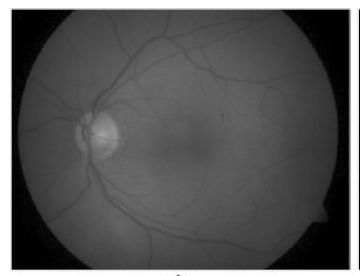

a)

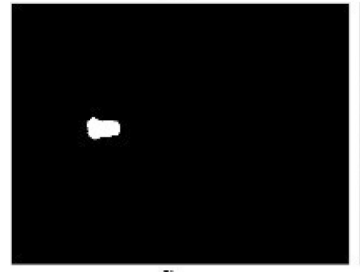

d)

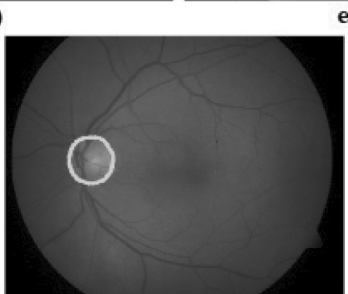

g)

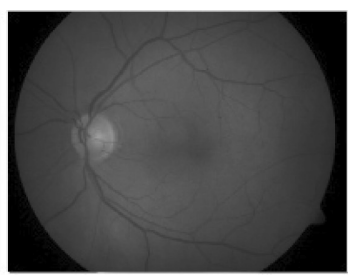

b)

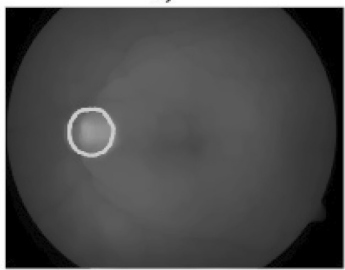

e)

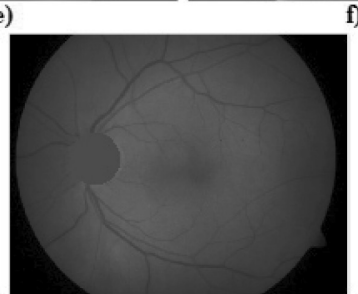

h)

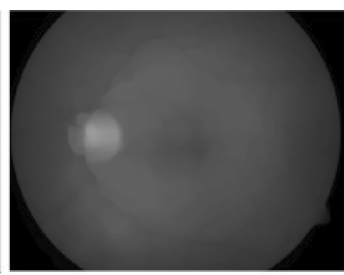

c)

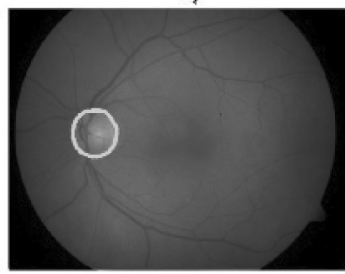

f)

Figure 9. (a) Original normal fundus. (b) Gray level fundus. (c) Preprocessed fundus. (d) Initial OD contour. (e) OD contour segmented with Greedy algorithm. (f) OD contour on original fundus. (g) OD contour on gray level fundus. (h) OD eliminated fundus. 


\section{EXPERIMENTAL RESULTS}

In this study, the classification is implemented via using both healthy and DR fundus images that include either soft exudates, hard exudates, or hemorrhages. The goal is to improve an automatic system, which separates DR fundus images from the healthy ones. The extracted features obtained from Curvelet Transform and SIFT are given as input to the SVM classifier. Fig. 10 represents OD eliminated fundus images and their corresponding curvelet coefficients. Classification stage of the proposed system is implemented in WEKA (Hall et al., 2009) with SVM. Weka provides machine learning algorithms for data mining tasks. It contains tools for data preprocessing, regression, classification, and clustering. In this study, polynomial kernel function is used for the SVM method, and the hyperparameter $c$ is chosen as 1.0. For each fundus image, 128 curvelet subband coefficients and 50 SIFT features are obtained.

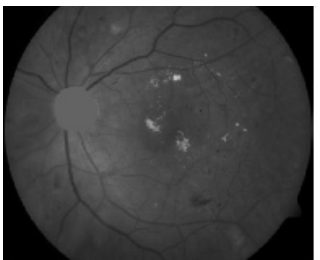

a)

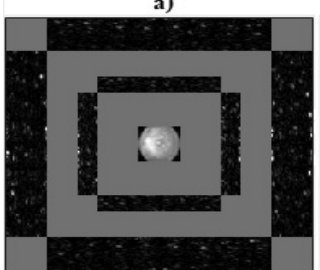

d)

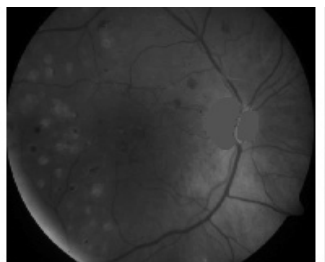

b)

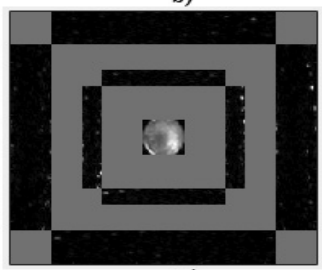

e)

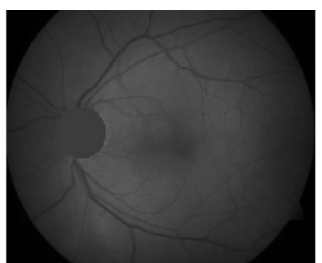

c)

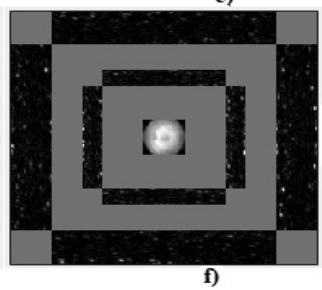

Figure 10. (a-c) OD eliminated fundus images. (d-f) Curvelet Transform coefficients of the corresponding images.

According to the experimental results, the classification accuracy (Acc.) is calculated as $92.8 \%$ using equation (21). Furthermore, the most commonly used performance metrics such as precision, recall (sensitivity or True Positive Rate (TPR)), False Positive Rate (FPR), F-measure, specificity (True Negative Rate (TNR)), and Matthews Correlation Coefficient (MCC) are obtained using equations (22)-(27), respectively. Also Kappa Statistics (KS), Mean Absolute Error (MAE), Root Mean Squared Error (RMSE) (Lavanya et al., 2020), and Area under ROC (AUC) metrics are calculated. True Positive (TP), False Positive (FP), False Negative (FN), and True Negative (TN) represent actual tumors, detected non-tumor ROIs, missed tumors, and correctly eliminated non-tumor ROIs, respectively.

$$
\begin{aligned}
& \text { Accuracy }=(T P+T N) /(T P+T N+F P+F N) \\
& \text { Precision }=\frac{T P}{T P+F P} \\
& \text { Recall }=\frac{T P}{T P+F N} \\
& F P R=\frac{F P}{F P+T N} \\
& F-\text { measure }=\frac{2 T P}{2 T P+F N+F P} \\
& \text { Specificity }=\frac{T N}{T N+F P} \\
& M C C=\frac{T P * T N-F P * F N}{[(T P+F P) *(F N+T N) *(F P+T N) *(T P+F N)]^{\frac{1}{2}}}
\end{aligned}
$$


For comparison, NB and KNN (Karamizadeh and Abdullah, 2018) classifiers are applied in the experiments whose results are demonstrated in Tables 1 and 2. SVM classifier produces the highest accuracy of $92.8 \%$ using the proposed methodology. KS value is calculated as 0.829 , precision is 0.922 , recall is 0.976 , and F-measure is 0.949 . Also AUC is 0.901 , specificity is 0.825 , and $\mathrm{MCC}$ is 0.833 . On the other hand, $\mathrm{KNN}$ classifier produces the highest specificity of 0.925. MAE, RMSE, and FPR column charts and values are seen in Fig. 11 and Table 3, respectively. Table 3 lists the confusion matrices of SVM, NB, and KNN. One can see the ROC curve of the SVM classifier with $92.8 \%$ accuracy in Fig. 12.

Table 1. Performance evaluators of the proposed method with SVM, NB, and KNN classifiers.

\begin{tabular}{ccccccccc}
\hline & KS & Precision & Recall & F-Measure & AUC & Specificity & MCC & $\begin{array}{c}\text { Acc. } \\
\text { (\%) }\end{array}$ \\
\hline SVM & 0.829 & 0.922 & 0.976 & 0.949 & 0.901 & 0.825 & 0.833 & 92.8 \\
NB & 0.788 & 0.902 & 0.976 & 0.938 & 0.876 & 0.775 & 0.795 & 91.2 \\
KNN & 0.772 & 0.962 & 0.882 & 0.920 & 0.970 & 0.925 & 0.778 & 89.6 \\
\hline
\end{tabular}

Table 2. RMSE, FPR, and MAE values of the proposed method with SVM, NB, and KNN classifiers.

\begin{tabular}{cccc}
\hline & RMSE & FPR & MAE \\
\hline SVM & 0.268 & 0.175 & 0.072 \\
NB & 0.297 & 0.225 & 0.088 \\
KNN & 0.321 & 0.075 & 0.104 \\
\hline
\end{tabular}

Table 3. Confusion matrix of SVM, NB, and KNN classifiers.

\begin{tabular}{ccccc}
\hline & $\begin{array}{c}\text { True } \\
\text { Positive }\end{array}$ & $\begin{array}{c}\text { True } \\
\text { Negative }\end{array}$ & $\begin{array}{c}\text { False } \\
\text { Positive }\end{array}$ & $\begin{array}{c}\text { False } \\
\text { Negative }\end{array}$ \\
\hline SVM & 83 & 33 & 7 & 2 \\
NB & 83 & 31 & 9 & 2 \\
KNN & 75 & 37 & 3 & 10 \\
\hline
\end{tabular}

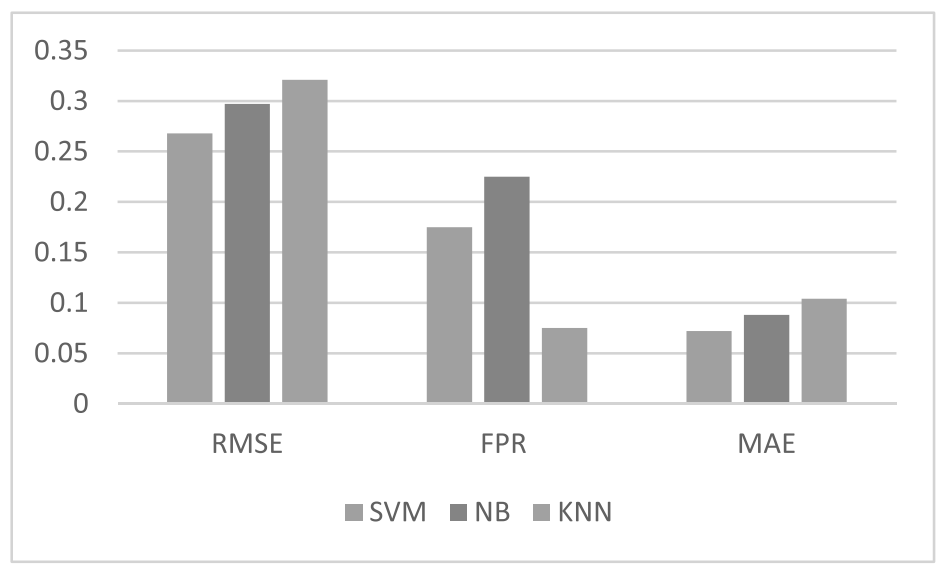

Figure 11. Column charts of RMSE, FPR, and MAE metrics. 


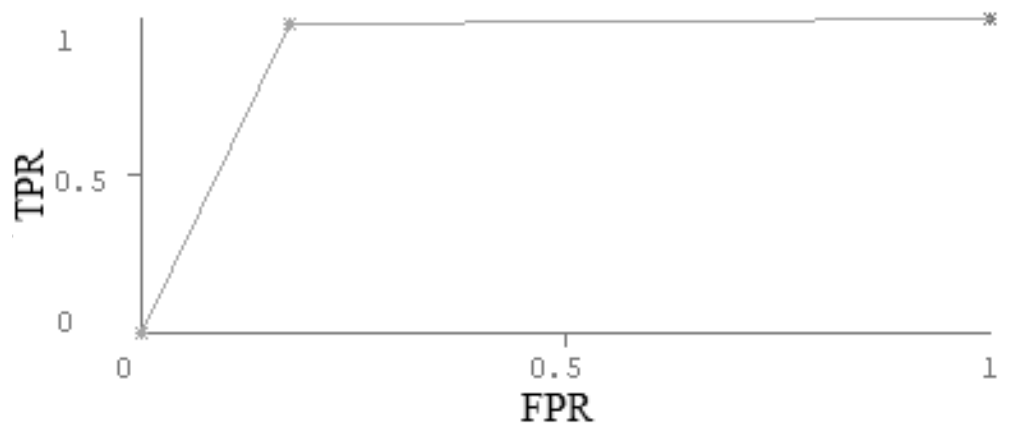

Figure 12. ROC curve of SVM.

The accuracy of the proposed work is compared against various existing approaches in the area of detection DR, and the results are highlighted in Table 4. It could be observed that the proposed method is advisable when compared to other methods.

Table 4. Comparison of classification accuracy.

\begin{tabular}{lll}
\hline & Method & Acc.(\%) \\
\hline Harangi and Hajdu (2014) & Active contour + NB & 82.0 \\
Zhu et al. (2019) & Morphological shape features + RF & 85.6 \\
Acharya et al. (2009) & Morphological texture features + SVM & 85.9 \\
Lin et al. (2018) & CNN & 86.1 \\
Yafen et al. (2013) & Texture homogeneity analysis + SVM & 89.0 \\
Ram and Sivaswamy (2009) & Multi-space features + K-Means & 89.6 \\
Osareh et al. (2002) & FCM & 90.1 \\
Gadekallu et al. (2020) & DNN + *PCA & 90.1 \\
Hua et al. (2019) & Skip-connection Deep Networks & 90.6 \\
Koh et al. (2017) & 2D-CWT+ FCM & 92.4 \\
Agurto et al. (2010) & AM-FM features & 92.0 \\
Li et al. (2019) & Novel deep network & 92.0 \\
Acharya et al. (2011) & HOS+SVM & 91.0 \\
Proposed Method & Greedy + CT + SIFT + SVM & $\mathbf{9 2 . 8}$ \\
\hline
\end{tabular}

*PCA: Principal Component Analysis.

\section{CONCLUSION}

Computer aided diagnosis methods provide efficient solutions for DR detection and classification. This study is a computer based system that distinguishes normal and DR fundus. The experimental results confirm the superiority of using the proposed methodology based on Greedy based OD segmentation, CT based feature extraction, and SIFT features. Firstly, morphological operations such as filtering, opening, and dilation are utilized to define the initial OD contours. Greedy algorithm uses these contours to segment the OD regions exactly. As OD regions may be thought to be DR signs due to their intensity, they are discarded before the feature extraction stage. Next, the features extracted 
by the curvelet transform and scale invariant feature transform are concatenated to provide a feature set that defines the fundus data optimally and contains important diagnostic information. The feature set is given to SVM, KNN, and NB to classify the fundus as normal or affected by DR. The proposed methodology can discriminate the DR with an accuracy of $92.8 \%$, sensitivity of $97.6 \%$, and specificity of $92.5 \%$. When compared with related work, this automatic identification approach has the potential to be used as a clinical helper in the diagnosis of DR, which can improve the diagnostic efficiency. And it can be concluded that the proposed methodology is effective in detecting DR abnormality accurately with suitable preprocessing for Greedy segmentation, CT decomposition, and SIFT features. In the future, the feature extraction step can be enhanced using deep learning classification models with additional curvelet based methods such as Shearlet transform. In this way, the performance of the proposed model can be further improved in terms of better and effective diagnosis.

\section{REFERENCES}

Abed, S., Al-Roomi, S.A. \& Al-Shayeji, M. (2016). Effective optic disc detection method based on swarm intelligence techniques and novel pre-processing steps. Applied Soft Computing. 49(2016):146-163.

Abed, S., Al-Oraifan, D. \& Safar, A. (2019). Optic disc detection using fish school search algorithm based on FPGA, Journal of Engg. Research, 7(3):161-177.

Acharya, U.R., Lim, C.M., Ng, E.Y.K., Chee, C. \& Tamura, T. (2009). Computer-based detection of diabetes retinopathy stages using digital fundus images. Proc. Inst. Mech. Eng. HJ. Eng. Med. 223(5): 545-553.

Acharya, U.R., Dua, S., Du, X., Sree, V.S. \& Chua, K.C. (2011). Automated diagnosis of glaucoma using texture and higher order spectra features. IEEE Trans. Inf. Technol. Biomed.: A Publ. IEEE Eng. Med. Biol. Soc. 15(3): 449-455.

Adem, K. (2018). Exudate detection for diabetic retinopathy with circular Hough transformation and convolutional neural networks. Expert Systems with Applications. 114: 289-295.

Agurto, C. et.al. (2010). Multiscale am-fm methods for diabetic retinopathy lesion detection. IEEE Trans Med Imaging. 29(2): 502-12. doi:10.1 109/TM1.2009.2037 146.

Alipour, S., H., M., Rabbani, H. \& Akhlaghi, M.R. (2012). Diabetic retinopathy grading by digital curvelet transform. Comput Math Methods Med. 2012: 1-11.

Alshayeji, M., Al-Roomi, S.A. \& Abed, S. (2017). Optic disc detection in retinal fundus images using gravitational law-based edge detection. Med Biol Eng Comput. 55: 935-948.

Asha, P.R. \& Karpagavalli, S. (2015). Diabetic retinal exudates detection using machine learning techniques. International Conference on Advanced Computing and Communication Systems. Kochi, India.

Candès, E.J. \& Donoho, D.L. (2000). Curvelets: a surprisingly effective nonadaptive representation of objects with edges in Curve and Surface Fitting. Saint-Malo 1999 Albert Cohen, Christophe Rabut, and Larry L. Schumaker (eds.) Vanderbilt University Press, Nashville, TN.

Colas, E., Besse, A., Orgogozo, A., Schmauch, B., Meric, N. \& Besse, E. (2016). Deep learning approach for diabetic retinopathy screening. Acta Ophthalmol. 94(S256).

Cortes, C. \& Vapnik, V. (1995). Support-Vector Networks. Machine Learning. 20: 273-297.

Decencière, E., Cazuguel, G., Zhang, X., Thibault, G., Klein, J-C., Meyer, F. et al. (2013). Teleophta: machine learning and image processing methods for teleophthalmology. Ingénierie et Recherche Biomédicale. 34(2) :196-203.

Doshi, D., Shenoy, A., Sidhpura, D. \& Gharpure, P. (2016). Diabetic retinopathy detection using deep convolutional neural networks. 2016 International Conference on Computing, Analytics and Security Trends (CAST). Pune, India.

Gadekallu, T.R., Khare, N., Bhattacharya, S., Singh, S. et al. (2020). Early detection of diabetic retinopathy using PCA-Firefly based deep learning model. Electronics. 9(274).

Gonzalez, R.C. \& Woods, R.E. (2002). Digital Image Processing (Second Edition). Prentice Hall, USA.

Görgel, P. \& Dincer, N. (2018). Computer Aided Brain Tumor Detection via Rule Based Eliminated Watershed Segmentation. 6th International Conference on Control Engineering \& Information Technology (CEIT). Istanbul, Turkey. 
Görgel, P. (2019). Brain Tumor Detection via Active Contours and Scale Invariant Feature Transform. ICATCES 2019 Proceeding Book International Conference on Advanced Technologies, Computer Engineering and Science 26-28 Apr 2019. Alanya, Turkey.

Gulshan, V., Peng, L., Coram, M., Stumpe, M.C., Wu, D., Narayanaswamy, A. et al. (2016). Development and validation of a deep learning algorithm for detection of diabetic retinopathy in retinal fundus photographs. JAMA. 316(22) : 2402-2410.

Hall, M., Frank, E., Holmes, G., Pfahringer, B., Reutemann, P. \& Witten, I.H. (2009). The WEKA Data Mining Software: An Update; SIGKDD Explorations, 11(1).

Harangi, B. \& Hajdu, A. (2014). Automatic exudates detection by fusing multiple active contours and region-wise classification. Computers in Biology and Medicine. 54:156-171.

Hua, C.H., Thien, H.T., Kim, K., Yu, S.Y. et al. (2019). Bimodal learning via trilogy of skip- connection deep networks for diabetic retinopathy risk progression identification. International Journal of Medical Informatics. 132(Article id: 103926).

Kauppi, T., Kalesnykiene, V., Kamarainen, J.K., Lensu, L., Sorri, I., Uusitalo, H. et al. (2006). DIARETDB0: evaluation database and methodology for diabetic retinopathy algorithms. Machine vision and pattern recognition research group p.73. Finland: Lappeenranta University of Technology.

Kauppi, T., Kalesnykiene, V., Kamarainen, J.K., Lensu, L., Sorri, I., Pietila, J. \& Uusitalo, H. (2007). DIARETDB1-standard diabetic retinopathy database. IMAGERET-Optimal detection and decision-support diagnosis of diabetic retinopathy.

Karaköse, M., Yetis, H. \& Müezzinoglu, T. (2017). Optimizasyon Tabanlı Adaptif Görüntü Mozaikleme Algoritması [Optimization Based Adaptive Image Pixelization Algorithm]. Bilişim Teknolojileri Dergisi, 10(4): 389-400.

Karamizadeh, S. \& Abdullah, S.M. (2018). Race classification using Gaussian-based weight K-NN algorithm for face recognition. Journal of Engineering Research. 6(2): 103-121.

Kass, M., Witkin, A. \& Terzopoulos, D. (1988). Snakes: Active Contour Models. International Journal of Computer Vision, 321331.

Koh, J.E.W., U. Acharya, R., Hagiwara, Y. et al. (2017). Diagnosis of retinal health in digital fundus images using continuous wavelet transform (CWT) and entropies. Computers in Biology and Medicine. 84: 89-97.

Lachure, J., Deorankar, A.V., Lachure, S., Swati, G. \& Jadhav, R. (2015). Diabetic retinopathy using morphological operations and machine learning. IEEE International Advance Computing Conference. Banglore, India.

Lavanya, P.V., Narasimhulu, C.V. \& Prasad, K.S. (2020). Dual stage bayesian network with dual-tree complex wavelet transformation for image denoising. Journal of Engg. Research. 8(1): 154-178.

Li, X., Shen, L., Shen, M., Tan, F. \& Qiu, C.S. (2019). Deep learning based early stage diabetic retinopathy detection using optical coherence tomography. Neurocomputing. 369: 134-144.

Lin, G.M., Chen, M.J., Yeh, C.H., Lin, Y.Y. et al. (2018). Transforming retinal photographs to entropy images in deep learning to improve automated detection for diabetic retinopathy. Journal of Ophthalmology. 2018(Article id: 2159702).

Lowe, D.G. (1999). Object recognition from local scale-invariant features. Proceedings of the International Conference on Computer Vision. 2: 1150-1157.

Lowe, D.G. (2004). Distinctive Image Features from Scale-Invirant Keypoints. International Journal of Computer Vision. 60(2): 91-110.

Mookiah, M., R., K., Acharya, U., R., Kuang, C., C., Min, L., C. et al. (2013). Computer-aided diagnosis of diabetic retinopathy: a review. Comput Biol Med. 43(12): 2136-55.

Mordvintsev, A. \& Abid, K. (2013). ‘OpenCV-Python Tutorials'. [Online]. Available: https://opencv-python-tutroals.readthedocs. io/en/latest/py_tutorials/py_feature2d/py_sift_intro/py_sift_intro.html [Accessed: 01- Apr- 2019].

Nadeem, S., Malik, M., Saad, M. \& Aqsa, R. (2019). Diabetic retinopathy techniques in retinal images: A review. Artificial Intelligence in Medicine. 97: 168-188.

Nayak, D.R., Dash, R., Majhi, B. \& Prasad, V. (2017). Automated pathological brain detection system: A fast discrete curvelet transform and probabilistic neural network based approach. Expert Systems with Applications. 88 : 152-164.

Nixon, M.S. \& Aguado, A.S. (2002). Feature Extraction and Image Processing, Woburn, MA, Oxford University Press. 
Osareh, A., Mirmehdi, M., Thomas, B.T. \& Markham, R. (2002). Classification and localisation of diabetic-related eye disease, in: Proceedings of the7th European Conference on Computer Vision-Part IV, ECCV'02. Berlin, Heidelberg.

Panchall, P.M., Panchal, S.R. \& Shah, K. (2013). A Comparison of SIFT and SURF. International Journal of Innovative Research in Computer and Communication Engineering. 1(2): 323-327.

Qin, J., Yang, J., Xue, B. \& Bu, F. (2012). Research on Image Registration and Mosaic Basedon Vector Similarity Matching Principle. Fifth International Symposium on Computational Intelligence and Design. Hangzhou, China.

Ram, K. \& Sivaswamy, J. (2009). Multi-space clustering for segmentation of Exudates in Retinal Colour Photographs. Conf. Proc. IEEE Eng Med Bioi Soc. 1437-40. doi: 10.1109/IEMBS.2009.5332911.

Sarwar, N., Gao, P., Seshasai, SR., Gobin, R., Kaptoge, S., Di Angelantonio et al. (2010). Diabetes mellitus, fasting blood glucose concentration, and risk of vascular disease: a collaborative meta-analysis of 102 prospective studies. Lancet. 375(9733) : 2215-2222.

Sayin, N., Kara, N. \& Pekel, G. (2015). Ocular complications of diabetes mellitus. World J Diabetes. 6(1): 92-108.

Shang, Y., Diao, Y.H. \& Li, C.M. (2008). Rotation Invariant Texture Classification Algorithm Based on Curvelet Transform and Svm. Proceedings of the Seventh International Conference on Machine Learning and Cybernetics. 12-15 July 2008. 30323036.

Shanthi, T. \& Sabeenian, R.S. (2019). Modified Alexnet architecture for classification of diabetic retinopathy images. Computers and Electrical Engineering. 76: 56-64.

Weiss, B. (2006). Fast median and bilateral filtering. ACM Transactions on Graphics (TOG). 25(3): 519-526.

Whiting, DR., Guariguata, L., Weil, C. \& Shaw, J. (2011). IDF diabetes atlas: global estimates of the prevalence of diabetes for 2011 and 2030. Diabetes Res Clin Pract. 94: 311-321.

Williams, D.J. \& Shah, M. (1992). A Fast Algorithm for Active Contours and Curvature Estimation, CVGIP: Image Understanding, 55(1): $14-26$.

Yafen, L. et.al. (2013). Automated Identification of Diabetic Retinopathy Stages Using Support Vector Machine. Proceeding of the $32^{\text {nd }}$ Chinese Control Conference, July 26-28. Xi'an, China.

Zhang, M. (2008). Blood Vessel Detection in Retinal Images and Its Application in Diabetic Retinopathy Screening. PhD thesis. College Station, TX, USA: Texas University. AAI3333795.

Zhu, C.Z., Hu, R., Zou, B.J. et al. (2019). Automatic diabetic retinopathy screening via cascaded framework based on imageand lesion-level features fusion. Journal of Computer Science and Technology. 34(6): 1307-1318. 